\title{
Clinical observations on the corneal thickness and the corneal endothelium in diabetes mellitus
}

\author{
N. BUSTED, ${ }^{1}$ T. OLSEN, ${ }^{1}$ AND O. SCHMITZ ${ }^{2}$ \\ From the 'Department of Ophthalmology, and ${ }^{2}$ Second University Clinic of Internal Medicine, \\ University of Aarhus, Aarhus Kommunehospital, Aarhus, Denmark
}

SUMMARY The corneal thickness was measured by pachometry and the corneal endothelium was photographed by specular microscopy in 81 insulin-dependent juvenile diabetic outpatients. The corneal thickness of a normal group, diabetics without and with proliferative retinopathy was (mean \pm SD): $0.527 \pm 0.028,0.544 \pm 0.028$, and $0.566 \pm 0.027 \mathrm{~mm}$, respectively $(2 \mathrm{p}<0.01)$. As revealed in the specular photomicrographs, minute folds in the endothelial layer were found in 13 of the diabetics versus 1 of the normal group $(2 p<0 \cdot 01)$. The cell density and the occurrence of dystrophic changes in the endothelium did not differ from those in normal persons. The augmented corneal thickness in the diabetic subjects is tentatively interpreted as minimal corneal swelling. It seemed to be present very early in the disease and may thus be one of the earliest clinically detectable changes of the diabetic eye.

In a diabetic patient visible lesions take years to develop. However, functional abnormalities may be detected long before anatomical changes are evident. Very early in the disease an increased permeability of the capillary endothelium to plasma proteins is found, ${ }^{1}$ and retinal vessels may leak fluorescein before any retinopathy is visible. ${ }^{23}$

Like the vascular endothelium the function of the corneal endothelium is to act as a cellular barrier. If corneal endothelial function is compromised, corneal hydration and consequently the corneal thickness will increase ${ }^{4}$ Both the corneal thickness and the corneal endothelium can be studied in vivo in man. Because of these considerations we found it of interest to study the corneal thickness and the corneal endothelium in diabetic patients.

\section{Subjects and methods}

A total of 81 insulin-dependent juvenile onset diabetic outpatients were included in the study. Age range was 15-75 years, mean 34 years. Duration of diabetes ranged from 3 months to 49 years, mean 15 years. Fifty-nine were outpatients from the medical clinic and 22 were outpatients from the eye clinic. The central corneal thickness was measured with a

Correspondence to Dr Thomas Olsen, Department of Ophthalmology, University of Aarhus, Aarhus Kommunehospital, DK-8000 Aarhus C. Denmark. modified Haag-Streit pachometer. ${ }^{5}$ The measuring procedure was end-to-end alignment of the optical section with a slit width of $0.030 \mathrm{~mm}$. Single determinations taken as the nearest $0.005 \mathrm{~mm}$ reading on the pachometer scale were used, the standard deviation of which (consecutive readings) has been found to be about $0.005 \mathrm{~mm} .{ }^{6}$ No correction was made for corneal curvature or nonlinearity of the pachometer. In order to obtain one measurement per subject the right and left thickness measurements were averaged, unless the reading on one side was discarded (see below). The central corneal endothelium was photographed with a noncontact specular microscope and the cell density was estimated as described earlier. ${ }^{7}$ The right and left cell density estimates were averaged unless the estimate on one side was discarded (see below). From the photomicrographs it was noted whether dystrophic changes or folds (seen as dark lines on the specular micrograph) were present in the endothelial layer.

After the corneal investigations the patients were subjected to slit-lamp and ophthalmoscopic examination. If fundus examination revealed exudates and red dots or blot haemorrages on one or both sides, the patient was grouped as a case with background retinopathy. If new vessel formation was revealed in one or both eyes, the patient was listed as a case with proliferative retinopathy. Intraocular pressure was measured with a Goldmann applanation tonometer. 
The readings were used directly without correction for corneal thickness. The corneal investigations on the one side and the rest of the ocular examinations on the other side were done by two independent observers (T.O. and N.B., respectively). All investigations were performed in the afternoon.

In the morning of the examination day the outpatients from the medical department had their fasting blood glucose concentration measured, and the insulin dosage was recorded. Information on other medical treatment and duration of diabetes was obtained. Eyes with ocular hypertension, with any disease unrelated to diabetes, or which had been subject to surgery were excluded from the series. Eyes which had received laser treatment were discarded from the endothelial cell density series. Thickness readings of laser treated eyes were discarded only if the post-treatment period was less than 2 weeks. Patients with renal insufficiency or on medical treatment for arterial hypertension were excluded from the study.

Control values for the corneal thickness determinations were obtained by measuring 49 normal students or members of the medical staff in the age range 20 to 50 years, mean 30 years.

The normal group for the specular microscopic examination comprised 67 normal subjects in the age range 15 to 65 years, mean 38 years, with no history of ocular disease or trauma, past or present, other than senile lens opacities. Some of these subjects had been included in the normal group previously reported. ${ }^{7}$

\section{Results}

Forty-seven patients had no retinopathy, 11 had background retinopathy, and 23 had proliferative retinopathy. No significant difference was found between the corneal thickness of those with background retinopathy as compared to those without any retinopathy. The corneal thickness of the normal group, diabetics without proliferative retinopathy and diabetics with proliferative retinopathy was (mean \pm SD): $0.527 \pm 0 \cdot 028,0 \cdot 544 \pm 0.028$, and $0.566 \pm$ 0.027 respectively $(2 \mathrm{p}<0.01$, by unpaired $t$ test) (Fig. 1). In 5 patients the proliferative changes were unilateral. In these patients the corneal thickness was $0.566( \pm 0.016)$ and $0.564( \pm 0.015) \mathrm{mm}$ on the side with proliferative changes and on the contralateral side respectively (not significant by paired $t$ test). Of a total of 16 patients 8 had received laser treatment on one side only. In these patients corneal thickness was $0.556( \pm 0.021)$ and $0.558( \pm 0.022) \mathrm{mm}$ on the treated and untreated sides respectively (not significant by paired $t$ test).

In Fig. 2 the corneal thickness has been plotted against the disease duration. No significant correla-
MM CORNEAL THICKNESS

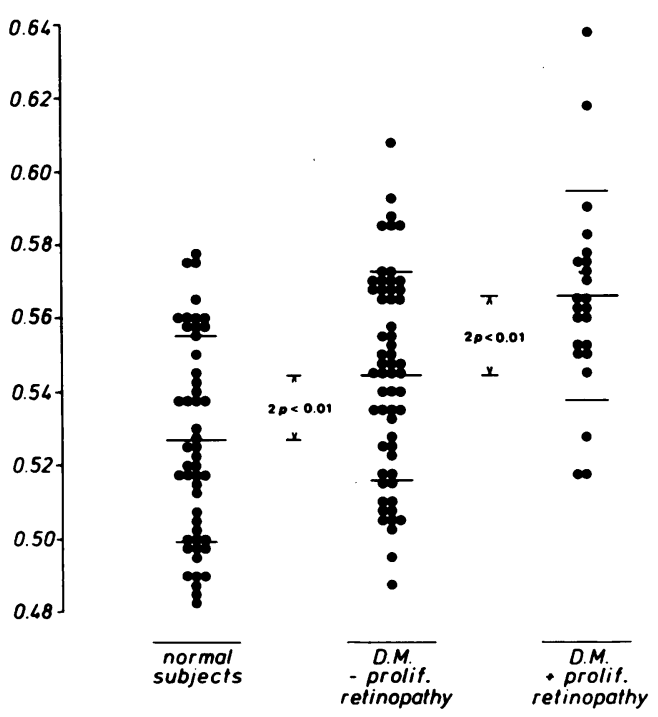

Fig. 1 Central corneal thickness in controls and in diabetics without or with proliferative retinopathy.

tion appeared. Also, no significant correlation was found between corneal thickness and fasting blood glucose or total daily insulin dosage. Mean fasting blood glucose concentration was $169 \mathrm{mg} / 100 \mathrm{ml}$ (range 55-336, $\mathrm{n}=53$ ) and $238 \mathrm{mg} / 100 \mathrm{ml}$ (range 128-331, $n=6$ ) in the group of diabetics without and with retinal proliferations. (SI conversion: $\mathrm{mmol} / \mathrm{l}=$ $\mathrm{mg} / 100 \mathrm{ml} \times 0.0555$.) Mean total daily insulin dosage was 45 IU (range 12-92) and 38 IU (range 28-48) in diabetics without and with retinal proliferations, respectively.

The normal endothelial cell density decreases with age. In Fig. 3 the age dependence of the diabetic group has been compared with the age dependence in the control group. Individual points are shown only for the diabetic group, but least-squares regression lines are shown for both the diabetic and the normal group. The slope of the 2 lines was not significantly different. The cell density correlated significantly with the duration of diabetes $(r=0 \cdot 28, n=73$, $2 p<0.05$ ). However, the decrease in cell density per year, as calculated from regression analysis was of the same magnitude as the normal age dependence (about 10 cells $/ \mathrm{mm}^{2}$ ). The mean cell density of those with and without proliferative retinopathy was $2686( \pm 356)$ and $2853( \pm 386)$ cells $/ \mathrm{mm}^{2}$, respectively $(2 \mathrm{p}=0.06)$. Central endothelial guttae were found in 11 patients, especially in the older age groups. In this the patients did not differ from the controls.

Folds in the central endothelial layer (Fig. 4) were 
MM CORNEAL THICKNESS

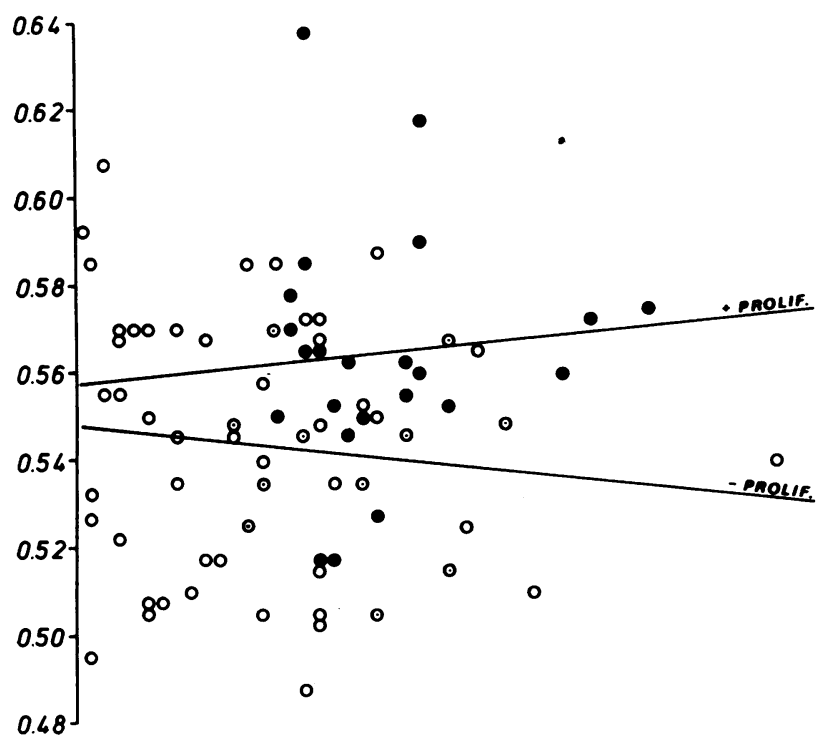

Fig. 2 Central corneal thickness in diabetics plotted against disease duration. Open circles: no retinopathy, circles with dot: background retinopathy, filled circles: proliferative retinopathy. Included are 2 regression lines calculated for diabetics without or with proliferative retinopathy respectively. No significant correlation is observed.

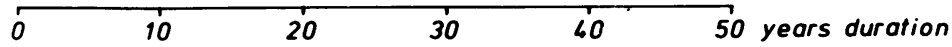

found in $13(16 \%)$ of the diabetics, significantly more frequently than in the normal subjects (Table 1). The mean corneal thickness of these subjects was 0.560 $( \pm 0.033) \mathrm{mm}$. Ten $(77 \%)$ of these patients had proliferative retinopathy.

Table 1 Prevalence of folds in the endothelium as revealed on specular micrographs of the central endothelium

\begin{tabular}{lll}
\hline & Number & With fold \\
\hline Diabetics & 81 & $13^{*}$ \\
Normal subjects & 67 & 1 \\
\hline
\end{tabular}

*2p $<0 \cdot 01$ by chi-square test.

The mean intraocular pressure in the diabetic group was $15.3( \pm 2 \cdot 4) \mathrm{mmHg}$. The tension was not found to correlate with the corneal thickness or to differ between diabetics with or without retinal complications.

\section{Discussion}

The increased corneal thickness in the diabetic patients may be due to either an increased water content or an increased dry weight content of the cornea or both. The results of the present study do not permit a definite judgment between these possibilities. However, the presence of small folds in
Descemet's membrane may be taken as evidence for an increased hydration of the cornea.

The presence of folds in Descemet's layer is a well known clinical feature of corneal oedema, and it

\section{CELLS $/ M^{2}$}

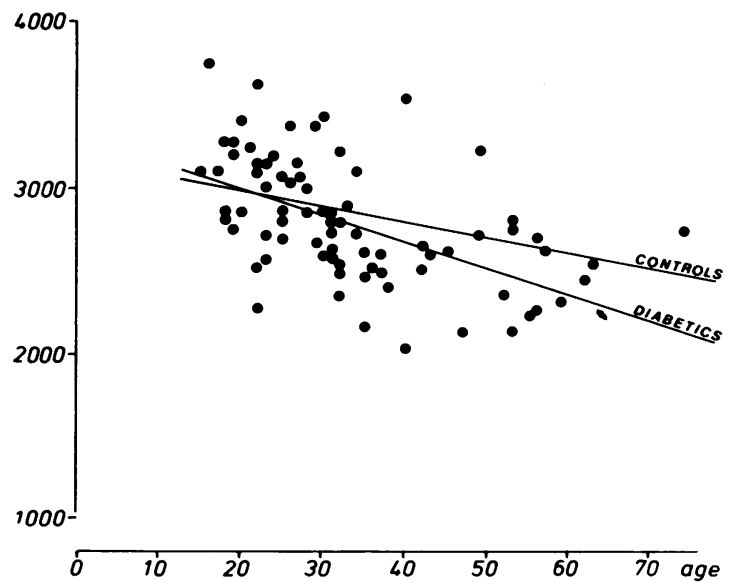

Fig. 3 Age-dependence of endothelial cell density for the diabetic subjects. Regression line $y=3322-15 \cdot 1 x$. Regression line for normal subjects is also shown. $y=3219-10 \cdot 6 x$. The slopes of the 2 lines are not statistically different $(2 p=0 \cdot 13)$. 
Fig. 4 Specular photomicrograph of the corneal endothelium in 2 diabetics without (top) and with (bottom, arrows) minute fold in the endothelial layer. Bars $=100 \mu \mathrm{m}$.

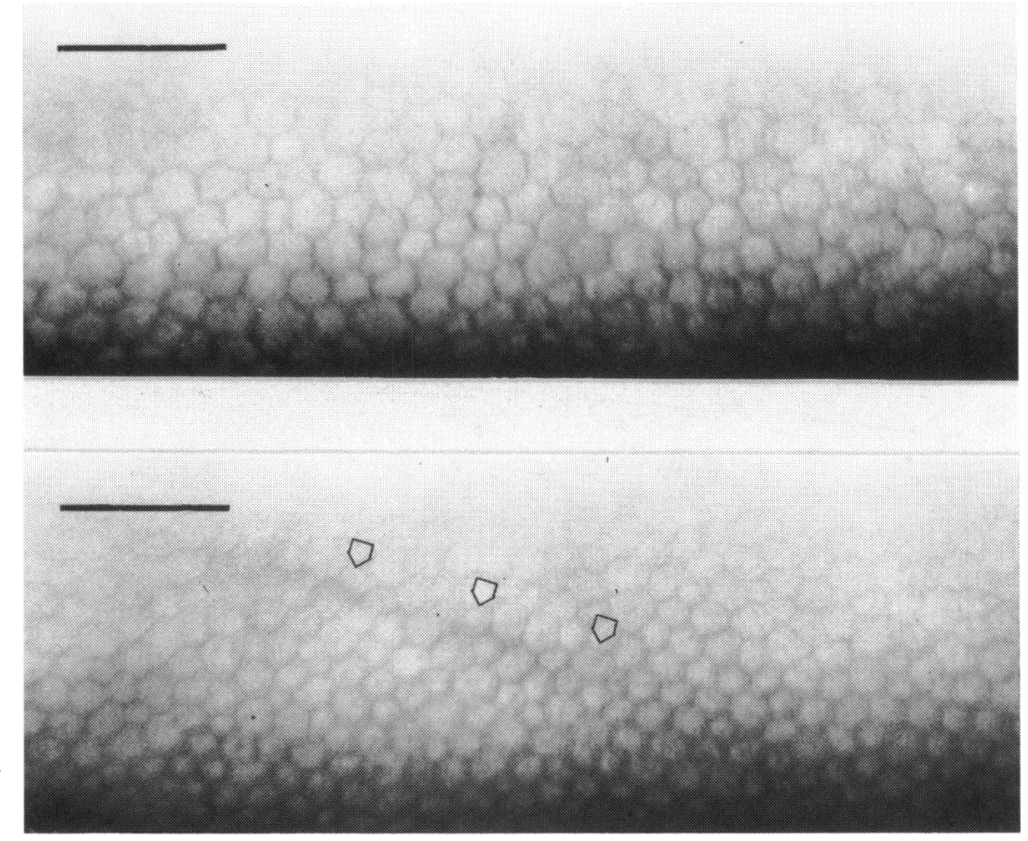

arises because the cornea can swell only in the posterior direction, thereby decreasing the area of the posterior corneal surface. The occurrence of minute folds in Descemet's membrane in diabetic patients has been reported by Waite and Beetham ${ }^{8}$ and Henkind and Wise. ${ }^{9}$ From slit-lamp examination of the whole cornea they found folds in 26 to $33 \%$ of the diabetic patients, especially in older persons. The lower prevalence found in the present study is probably due to the facts that only the central endothelium was examined and that the mean age was lower than in the above mentioned studies.

If the increased corneal thickness of the diabetics is due to an increased hydration of the cornea, an abnormal function of the corneal endothelium might be postulated as a cause. The morphology of the corneal endothelium in terms of the cell density was not found to differ significantly from normal and thus does not support this speculation. It should be noted, however, that the large scatter makes it difficult to assess a possible difference based on populations of cell counts.

The augmented corneal thickness seemed to be present very early in the disease and may thus be one of the earliest detectable changes of the diabetic eye. The association between increased corneal thickness and severe retinal complications suggests that the corneal thickness may be an indicator of the risk of retinal complications in diabetic individuals. This hypothesis is now investigated.

This study was supported by grants from the Danish Medical Research Council. The technical assistance of Mrs Anette Poulsen is gratefully acknowledged. A preliminary result of this study has been published. ${ }^{10}$

\section{References}

1 Parving H-H, Noer I, Deckert T, et al. The effect of metabolic regulation on microvascular permeability to small and large molecules in short-term juvenile diabetics. Diabetologia 1976; 12: 161-6.

2 Cunha-Vaz J, De Abreu JRF, Campos A. Figo GM. Early breakdown of the blood-retinal barrier in diabetes. $\mathrm{Br} \mathrm{J}$ Ophthalmol 1975; 59: 649-56.

3 Krupin T, Waltman SR, Oestrich C, et al. Vitreous fluorophotometry in juvenile-onset diabetes mellitus. Arch Ophthalmol 1978; 96: 812-4.

4 Mishima S. Corneal thickness. Surv Ophthalmol 1968; 13: 57-96.

5 Ehlers N. Sperling S. A technical improvement of the Haag-Streit pachometer. Acta Ophthalmol (Kbh) 1977; 55: 333-6.

6 Olsen T, Nielsen CB. Ehlers N. On the optical measurement of corneal thickness. II. The measuring conditions and sources of error. Acta Ophthalmol (Kbh) 1980; 58: 975-84.

7 Olsen T. Non-contact specular microscopy of human corneal endothelium. Acta Ophthalmol (Kbh) 1979; 57: 986-98.

8 Waite $\mathrm{JH}$, Beetham WP. The visual mechanism in diabetes mellitus. N Engl J Med 1935; 212: 367-429.

9 Henkind P. Wise GN. Descemet's wrinkles in diabetes. Am J Ophthalmol 1961; 52: 371-4.

10 Olsen T. Busted N. Schmitz O. Corneal thickness in diabetes mellitus. Lancet 1980; i: 883. 